\title{
Propagation Mechanism of Phase-Inversion Wave in 2D Lattice Oscillators and their Application to Prediction of Time-Series Data
}

\author{
Hitoshi ABURATANI, Suguru YAMANE, \\ Takeo IMOTO and Masayuki YAMAUCHI \\ Department of Electronics and Computer Engineering, \\ Hiroshima Institute of Technology \\ 2-1-1, Miyake, Saeki-ku, Hiroshima, Japan \\ Email: ab.hitoshi247@gmail.com
}

\begin{abstract}
In our previous study, we discovered phaseinversion waves on the lattice shape system. The phase-inversion waves are phenomena changing phase states between adjacent oscillators from in-phase to anti-phase or from anti-phase to in-phase and continuously. The phase-inversion waves can be observed in steady states. In this study, we observe and analyze propagation mechanisms of the phase-inversion waves in the lattice shape system. Furthermore, we predict air temperature data by using the lattice shape system.
\end{abstract}

\section{INTRODUCTION}

In this world, many kind of synchronization phenomenon can be observed. For example, we can observe the motions of many planets, schools of sardines, the synchronization of fireflies, and so on. The synchronization phenomenon is a field which catches interest of many researchers. Then, the studies of synchronization phenomena are researched in various fields[1],[2]. The synchronization phenomenon can be observed on coupled oscillators system[3]. We are investigating the synchronization phenomena on coupled oscillators system, and are developing some systems using synchronization phenomena.

In our previous study, we observed synchronization phenomena on the ladder shape systems and lattice shape systems. On the ladder shape systems, the phase-waves, which are phenomena of propagating a phase difference between adjacent oscillators, and the phase-inversion waves, which is a phenomenon of propagating a phase state between adjacent oscillators, were discovered. The propagation and reflection mechanisms of the phase-waves and the phase-inversion waves were clarified[4],[5]. On the lattice shape system, the phasewaves and the phase-inversion waves were discovered[6],[7]. Propagation and reflection mechanisms of the phase-waves were clarified[6]. Moreover, we developed a prediction method of time-series data using the lattice shape system. The timeseries data of Chua's circuit was predicted by this system[8].

In this study, we clarify propagation mechanisms of the phase-inversion wave using instantaneous frequency of each oscillator and phase differences between adjacent oscillators on the lattice shape system. Furthermore, the air temperature data is predicted by the lattice shape system. The air temperature data is one of many natural data.

\section{CIRCUIT MODEL}

A lot of van der Pol oscillators are coupled by inductors $L_{0}$ as a lattice(see Fig. 1). The number of column and the number of row of this system are assumed as 20 respectively. We name each oscillator $\operatorname{OSC}(k, l)$. A voltage of each oscillator is named $v_{(k, l)}$, and a current of inductor of each oscillator is

\author{
Yoshifumi NISHIO \\ Department of Electrical and Electronic Engineering, \\ Tokushima University \\ 2-1 Minami-Josanjima, Tokushima, Japan
}

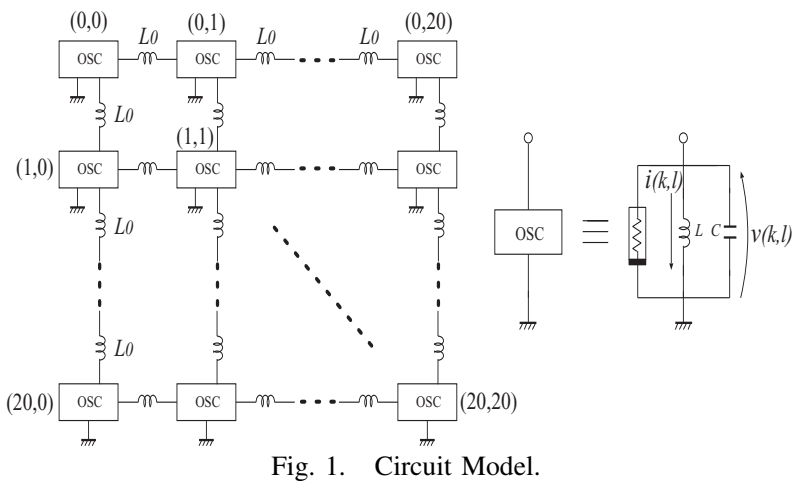

named $i_{(k, l)}$ (see Fig. 1). The circuit equations of this circuit model are normalized by Eq. (1), and the normalized circuit equations are shown as Eqs. (2)-(6).

$$
\begin{aligned}
& i_{(k, l)}=\sqrt{\frac{C g_{1}}{3 L g_{3}}} x_{(k, l)}, \quad v_{(k, l)}=\sqrt{\frac{g_{1}}{3 g_{3}}} y_{(k, l)}, \\
& t=\sqrt{L C} \tau, \quad \frac{d}{d \tau}=\cdots . ", \alpha=\frac{L}{L_{0}}, \quad \varepsilon=g_{1} \sqrt{\frac{L}{C}} .
\end{aligned}
$$

[Corner-top] (left: $(a, b)=(0,1)$. right: $(a, b)=(19,18)$.)

$$
\begin{aligned}
& \frac{d x_{(0, a)}}{d \tau}=y_{(0, a)}, \\
& \begin{aligned}
\frac{d y_{(0, a)}}{d \tau}=-x_{(0, a)}+\alpha\left(x_{(0, b)}+x_{(1, a)}\right. & \left.-2 x_{(0, a)}\right) \\
& +\varepsilon\left(y_{(0, a)}-\frac{1}{3} y_{(0, a)}^{3}\right) .
\end{aligned}
\end{aligned}
$$

[Corner-bottom] (left: $(a, b)=(0,1)$. right: $(a, b)=(19,18)$.)

$$
\begin{aligned}
\frac{d x_{(19, a)}}{d \tau}=y_{(19, a)} & \\
\frac{d y_{(19, a)}}{d \tau}=-x_{(19, a)} & +\alpha\left(x_{(18, a)}+x_{(19, b)}\right. \\
& \left.-2 x_{(19, a)}\right)+\varepsilon\left(y_{(19, a)}-\frac{1}{3} y_{(19, a)}^{3}\right) .
\end{aligned}
$$

[Center] $(0<k<19.0<l<19$. $)$

$$
\begin{aligned}
& \frac{d x_{(k, l)}}{d \tau}=y_{(k, l)}, \\
& \frac{d y_{(k, l)}}{d \tau}=-x_{(k, l)}+\alpha\left(x_{(k+1, l)}+x_{(k-1, l)}+x_{(k, l+1)}+x_{(k, l-1)}\right. \\
& \left.-4 x_{(k, l)}\right)+\varepsilon\left(y_{(k, l)}-\frac{1}{3} y_{(k, l)}^{3}\right) .
\end{aligned}
$$

$[$ Edge] $($ top: $(a, b)=(0,1) \cdot$ bottom: $(a, b)=(19,18) \cdot$ both: $0<l<19$. $)$

$$
\begin{aligned}
& \frac{d x_{(a, l)}}{d \tau}=y_{(a, l)}, \\
& \begin{aligned}
\frac{d y_{(a, l)}}{d \tau}=-x_{(a, l)}+\alpha\left(x_{(a, l-1)}+x_{(a, l+1)}\right. & \left.+x_{(b, l)}-3 x_{(a, l)}\right) \\
+ & \varepsilon\left(y_{(a, l)}-\frac{1}{3} y_{(a, l)}^{3}\right) .
\end{aligned}
\end{aligned}
$$


[Edge] $($ left: $(a, b)=(0,1)$. right: $(a, b)=(19,18)$. both: $0<k<19$. $)$

$$
\begin{aligned}
& \frac{d x_{(k, a)}}{d \tau}=y_{(k, a)} \\
& \begin{aligned}
\frac{d y_{(k, a)}}{d \tau} & =-x_{(k, a)}+\alpha\left(x_{(k-1, a)}+x_{(k+1, a)}\right. \\
& +x_{(k, b)}-3 x_{(k, a)} \\
& +\varepsilon\left(y_{(k, a)}-\frac{1}{3} y_{(k, a)}^{3}\right) .
\end{aligned}
\end{aligned}
$$

The $\alpha$ corresponds to the coupling parameter of each oscillator. The $\varepsilon$ corresponds to the nonlinearity of each oscillator. This system is simulated by the fourth order Runge-Kutta methods using Eqs. (2)-(6).

\section{PROPAGATION MECHANISMS}

A propagation of a phase-inversion waves can be observed(see Fig.2). The propagation mechanisms are made clear using frequency of each oscillator and phase difference between adjacent oscillators. The coupling parameter is fixed as $\alpha=0.01$, and nonlinearity is fixed $\varepsilon=0.050$.

An equation of the instantaneous frequency of $\operatorname{OSC}(k, l)$ is calculated as follows. The instantaneous frequency is named $f_{(k, l)}(a)$ where "a" expresses the number of times of the peak value of the voltage. Time of a peak value of the voltage of $\operatorname{OSC}(k, l)$ is assumed as $\tau_{(k, l)}(a)$ (see Fig.3). Similarly, $\tau_{(k+1, l)}(a)$ and $\tau_{(k, l+1)}(a)$ are decided. The $f_{(k, l)}(a)$ is obtained by Eq.(7).

$$
f_{(k, l)}(a)=\frac{1}{\tau_{(k, l)}(a)-\tau_{(k, l)}(a-1)} .
$$

Three frequencies are observed in steady states. In this system, the synchronizations for vertical direction and horizontal direction needs to be considered, because this system is 2 dimensional array. Therefore, three type synchronizations are observed as follows:

1. $\operatorname{OSC}(k, l)-\operatorname{OSC}(k, l+1), \operatorname{OSC}(k, l)-\operatorname{OSC}(k, l-1), \operatorname{OSC}(k$, $l)-\operatorname{OSC}(k+1, l)$, and $\operatorname{OSC}(k, l)-\operatorname{OSC}(k-1, l)$ : in-phase synchronization.

2. $\{(\operatorname{OSC}(k, l)-\mathrm{OSC}(k, l+1)$ and $\operatorname{OSC}(k, l)-\mathrm{OSC}(k, l-1)$ : inphase synchronization. $\operatorname{OSC}(k, l)-\operatorname{OSC}(k+1, l)$, and $\operatorname{OSC}(k$, $l)-\operatorname{OSC}(k-1, l)$ : anti-phase synchronization. $\}$ or $\{\operatorname{OSC}(k$, $l)-\operatorname{OSC}(k, l+1)$, and $\operatorname{OSC}(k, l)-\operatorname{OSC}(k, l-1)$ : anti-phase synchronization. $\operatorname{OSC}(k, l)-\operatorname{OSC}(k+1, l)$, and $\operatorname{OSC}(k, l)-$ $\operatorname{OSC}(k+1, l)$ : int-phase synchronization. $\}$

3. $\operatorname{OSC}(k, l)-\operatorname{OSC}(k, l+1), \operatorname{OSC}(k, l)-\operatorname{OSC}(k, l-1), \operatorname{OSC}(k$, $l)-\operatorname{OSC}(k+1, l)$, and $\operatorname{OSC}(k, l)-\operatorname{OSC}(k+1, l)$ : anti-phase synchronization.

In this paper, we call the 1st type synchronization "in-and-inphase synchronization." The 2nd type synchronization is called "in-and-anti-phase synchronization." The 3rd type synchronization is called "anti-and-anti-phase synchronization." An each instantaneous frequency of $\operatorname{OSC}(k, l)$ is obtained in each synchronization type. In the 1st situational synchronization, $f_{(k, l)}$ is $f_{i n-i n}$. In the 2nd situational synchronization, $f_{(k, l)}$ is $f_{\text {in-anti }}$. In the 3 rd situational synchronization, $f_{(k, l)}$ is $f_{\text {anti-anti }}$.

The phase difference is calculated as follows. A phase difference between $\operatorname{OSC}(k, l)$ and $\operatorname{OSC}(k+1, l)$ and a phase difference between $\operatorname{OSC}(k, l)$ and $\operatorname{OSC}(k, l+1)$ are calculated. The phase differences are assumed as $\Phi_{(k, l)(k+1, l)}(a)$ and $\Phi_{(k, l)(k, l+1)}(a)$ respectively(see Fig.3). The $\Phi_{(k, l)(k+1, l)}(a)$ and $\Phi_{(k, l)(k, l+1)}(a)$ are obtained by Eq. $(8)$.

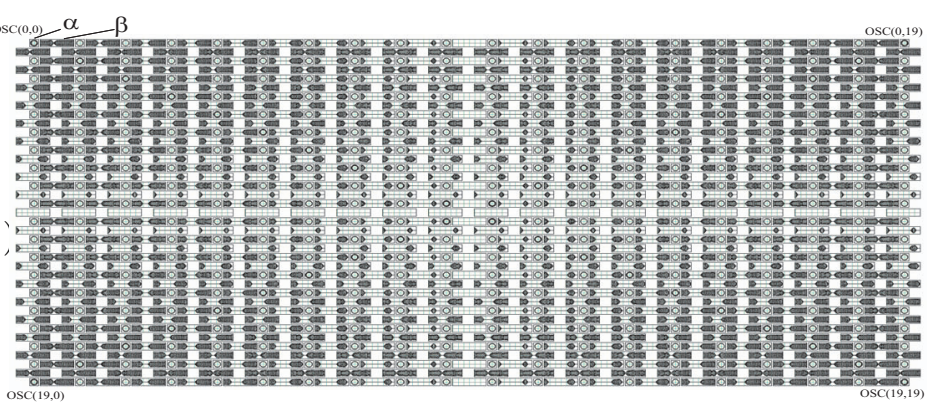

Fig. 2. The phase-inversion waves on $20 \times 20$ oscillators $(\alpha$ :an attractor of each oscillator(current vs. voltage), $\beta$ : a sum of voltages of adjacent oscillators(sum of voltage vs. time)).

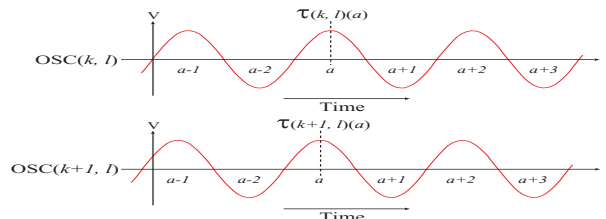

Fig. 3. The detection method of frequencies and the phase differences.

$$
\begin{aligned}
& \Phi_{(k, l)(k+1, l)}(a)=\frac{\tau_{(k, l)}(a)-\tau_{(k+1, l)}(a)}{\tau_{(k, l)}(a)-\tau_{(k, l)}(a-1)} \times 180 \text { [degree] } \\
& \Phi_{(k, l)(k, l+1)}(a)=\frac{\tau_{(k, l)}(a)-\tau_{(k, l+1)}(a)}{\tau_{(k, l)}(a)-\tau_{(k, l)}(a-1)} \times 180 \text { [degree]. }
\end{aligned}
$$

The phase-inversion waves are generated by the following method. 1. Voltages and currents of all oscillators are set as same value, in other words all oscillators are set as in-phase synchronization. 2 . The signs of $x_{(k, l)}$ and $y_{(k, l)}$ of arbitrary oscillators are inverted instantaneously.

\section{A. Propagation mechanism I (propagation of phase-inversion waves in in-and-in-phase synchronizations)}

We can observe that a phase-inversion wave for a vertical direction in each column propagate in in-and-in-phase synchronization. A mechanism is shown as Tab.I. A mechanism of a phase-inversion wave for a horizontal direction can be explained same method. In Fig.4(a), the vertical axis is the instantaneous frequency, and the horizontal axis is time. In Fig.4(b), the vertical axis expresses the phase difference and the horizontal axis expresses time.
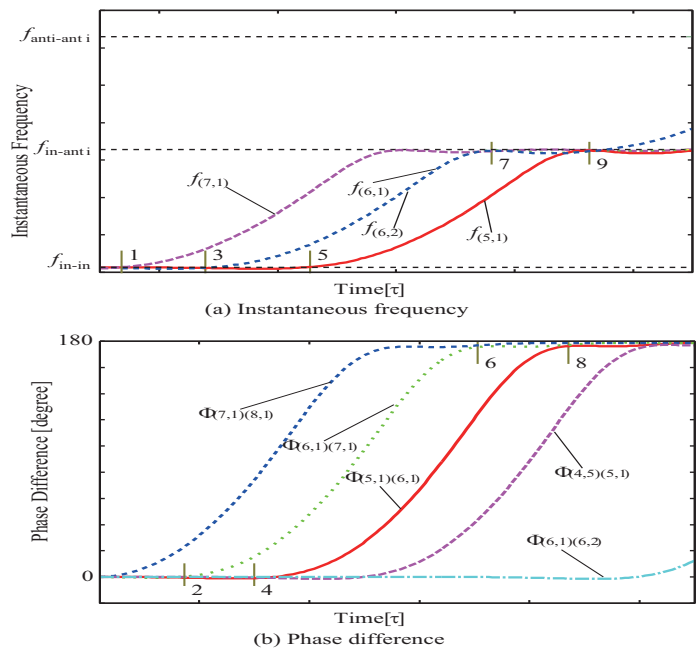

Fig. 4. Transitions of phase differences and frequencies by propagating a phase-inversion wave in in-and-in-phase synchronization. 
TABLE I

PROPAGATION MECHANISM OF A PHASE-INVERSION WAVE IN IN-AND-IN-PHASE SYNCHRONIZATION(SEE FIG.4).

\begin{tabular}{|c|c|}
\hline no. & Mechanism \\
\hline 0 & $\begin{array}{l}\Phi_{(8,0)(8,1)}, \Phi_{(8,1)(8,2)}, \Phi_{(7,0)(7,1)}, \Phi_{(7,1)(7,2)}, \Phi_{(6,0)(6,1)}, \\
\Phi_{(6,1)(6,2)}, \Phi_{(5,0)(5,1)} \text { and } \Phi_{(5,1)(5,2)} \text { are around } 0 \text { degrees } \\
\text { respectively. Then, a phase-inversion wave propagates for the ver- } \\
\text { tical direction in in-and-in-phase synchronization. Now, the phase- } \\
\text { ineversion wave, which changes from in-phase synchronization to } \\
\text { anti-phase synchronization, arrives at OSC }(4,7) .\end{array}$ \\
\hline 1 & $\begin{array}{l}f_{(7,1)} \text { starts to increase from } f_{i n-i n} \text { to } f_{i n-a n t i} \text {, because } \\
\Phi_{(7,1)(8,1)} \text { is changed from } 0 \text { degree to } 180 \text { degrees by a phase- } \\
\text { inversion wave. }\end{array}$ \\
\hline 2 & $\begin{array}{l}\Phi_{(6,1)(7,1)} \text { starts to increase from } 0 \text { degree to } 180 \text { degrees, because } \\
f_{(7,1)} \text { changes from } f_{i n-i n} \text { to } f_{i n-a n t i} .\end{array}$ \\
\hline 3 & $\begin{array}{l}f_{(6,1)} \text { starts to increase from } f_{i n-i n} \text { to } f_{i n-a n t i} \text { because } \\
\Phi_{(6,1)(7,1)} \text { increases toward } 180 \text { degrees. Instantaneous frequency } \\
f_{(6,2)} \text { of an adjacent oscillator starts to increase at same time } \\
\text { by propagating a phase-inversion wave in the adjacent column. } \\
\text { Therefore, } \Phi_{(6,1)(6,2)} \text { is fixed as around } 0 \text { degrees. The phase states } \\
\text { of both sides are the same motion. }\end{array}$ \\
\hline 4 & $\begin{array}{l}\Phi_{(5,1)(6,1)} \text { starts to increase from } 0 \text { degree to } 180 \text { degrees, because } \\
f_{(6,1)} \text { increases from } f_{\text {in-anti }} \text { to } f_{\text {anti-anti }} .\end{array}$ \\
\hline 5 & $\begin{array}{l}f_{(5,1)} \text { starts to increase from } f_{\text {in-anti }} \text { to } f_{\text {anti-anti }} \text {, because } \\
\Phi_{(5,1)(6,1)} \text { increased from } 0 \text { degree to } 180 \text { degrees. }\end{array}$ \\
\hline 6 & $\begin{array}{l}\Phi_{(6,1)(7,1)} \text { becomes around } 180 \text { degrees, and a phase state between } \\
\operatorname{OSC}(6,1) \text { and } \operatorname{OSC}(7,1) \text { is fixed anti-phase synchronization. }\end{array}$ \\
\hline 7 & $\begin{array}{l}f_{(6,1)} \text { arrives around } f_{\text {in }- \text { anti }} \text {, because the phase state between } \\
\operatorname{OSC}(6,1) \text { and } \operatorname{OSC}(7,1) \text { is anti-phase synchronization. }\end{array}$ \\
\hline 8 & $\begin{array}{l}\Phi_{(5,1)(6,1)} \text { arrives around } 180 \text { degrees, and a phase state between } \\
\text { OSC }(5,1) \text { and } \operatorname{OSC}(6,1) \text { is fixed anti-phase synchronization. }\end{array}$ \\
\hline 9 & $\begin{array}{l}f_{(5,1)} \text { arrives around } f_{\text {anti-anti }} \text {, because the phase state between } \\
\operatorname{OSC}(5,1) \text { and } \operatorname{OSC}(6,1) \text { is anti-phase synchronization. }\end{array}$ \\
\hline
\end{tabular}

B. Propagation mechanism II(propagating phase-inversion waves in in-and-anti-phase synchronizations)

The phase states of adjacent oscillators of horizontal directions are anti-phase synchronization, and the phase states of adjacent oscillators of vertical directions are in-phase synchronization. We can observe that a phase-inversion wave for a vertical direction in each column propagates in in-andanti-phase synchronization. A mechanism is shown as Tab.II. A mechanism of a phase-inversion wave for a horizontal direction can be explained same method. In Fig.5(a), the vertical axis is the instantaneous frequency, and the horizontal axis is time. In Fig.5(b), the vertical axis is the phase difference and the horizontal axis is time.

\section{APPLICATION FOR PREDICTION USING COUPLED OSCILLATORS SYSTEM}

We predict time-series data using the above system. Some parts of the system are changed something for the prediction system. 1. The number of this system's oscillators is fixed nine. 2. 8 inductors $\left(L_{\text {out }(k, l)}\right)$ connecting to an arbitrary signal generator are added to 8 oscillators of edges respectively, because arbitrary time-series data is input from outside(see Fig.6). Therefore, this system has 17 inductors, 9 capacitors and 9 nonlinear-negative-resistors. 3. These circuit elements are adjusted independently.

\section{A. Prediction method}

The original time-series data is assumed as $f(t)$. A prediction of the original time-series data is assumed as $g(t)$. The original time-series data of continuous data are divided by
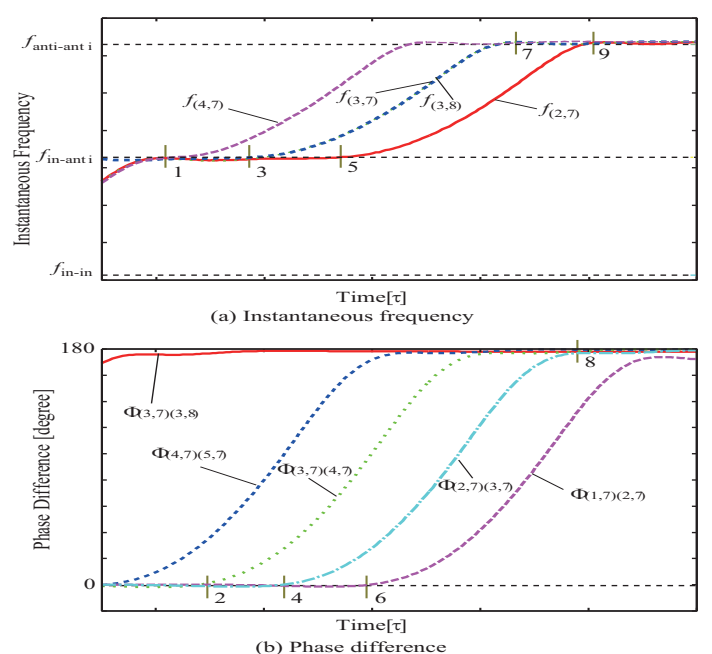

Fig. 5. Transitions of phase differences and frequencies by propagating a phase-inversion wave in in-and-anti-phase synchronization.

TABLE II

Propagation MECHANISM OF A PHASE-INVERSION WAVE IN IN-AND-ANTI-PHASE SYNCHRONIZATION(SEE FIG.5)

\begin{tabular}{|c|c|}
\hline no. & Mechanism \\
\hline 0 & $\begin{array}{l}\Phi_{(5,6)(5,7)}, \Phi_{(5,7)(5,8)}, \Phi_{(4,6)(4,7)}, \Phi_{(4,7)(4,8)}, \Phi_{(3,6)(3,7)} \text {, } \\
\Phi_{(3,7)(3,8)}, \Phi_{(2,6)(2,7)}, \Phi_{(2,7)(2,8)}, \Phi_{(1,6)(1,7)} \text { and } \Phi_{(1,7)(1,8)} \\
\text { are around } 180 \text { degrees respectively. Then, a phase-inversion wave } \\
\text { propagates for a vertical direction in in-and-anti-phase synchro- } \\
\text { nization, that the phase states between adjacent oscillators of } \\
\text { row direction are steadied as anti-phase synchronization and the } \\
\text { phase states between adjacent oscillators of column direction are } \\
\text { steadied as in-phase synchronization. Now, the phase-ineversion } \\
\text { wave, which changes from in-phase synchronization to anti-phase } \\
\text { synchronization, arrives at OSC }(4,7) \text {. }\end{array}$ \\
\hline 1 & $\begin{array}{l}f_{4,7} \text { starts to increase from } f_{\text {in-anti }} \text { to } f_{\text {anti-anti }} \text {, because } \\
\Phi_{(4,7)(5,7)} \text { ischanged from } 0 \text { degree to } 180 \text { degrees by a phase- } \\
\text { inversion wave. }\end{array}$ \\
\hline 2 & $\begin{array}{l}\Phi_{(3,7)(4,7)} \text { starts to increase from } 0 \text { degree to } 180 \text { degrees, because } \\
f_{(4,7)} \text { is changing toward } f_{\text {anti-anti }} \text {. }\end{array}$ \\
\hline 3 & $\begin{array}{l}f_{(3,7)} \text { starts to increase from } f_{\text {in-anti }} \text { to } f_{\text {anti-anti }} \text {, because } \\
\Phi_{(3,7)(4,7)} \text { is changed from } 0 \text { degree. Instantaneous frequency } \\
f_{(3,8)} \text { of an adjacent oscillator starts to increase at same time } \\
\text { by propagating a phase-inversion wave in the adjacent column. } \\
\text { Therefore, } \Phi_{(3,7)(3,8)} \text { is fixed as around } 180 \text { degrees. }\end{array}$ \\
\hline 4 & $\begin{array}{l}\Phi_{(2,7)(3,7)} \text { starts to increase from } 0 \text { degree to } 180 \text { degrees, because } \\
f_{(3,7)} \text { increases from } f_{i n-a n t i} \text { to } f_{\text {anti-anti }} \text {. }\end{array}$ \\
\hline 5 & $\begin{array}{l}f_{(2,7)} \text { starts to increase from } f_{\text {in-anti }} \text { to } f_{\text {anti-anti }} \text {, because } \\
\Phi_{(2,7)(3,7)} \text { increased from } 0 \text { degree to } 180 \text { degrees. }\end{array}$ \\
\hline 6 & $\begin{array}{l}\Phi_{(1,7)(2,7)} \text { starts to increase from } 0 \text { degree to } 180 \text { degrees, because } \\
f_{(2,7)} \text { starts to increase from } f_{i n-a n t i} \text { to } f_{\text {anti-anti }} \text {. }\end{array}$ \\
\hline 7 & $\begin{array}{l}f_{(3,7)} \text { arrives around } f_{\text {anti-anti }} \text {, because the phase state between } \\
\operatorname{OSC}(3,7) \text { and } \operatorname{OSC}(4,7) \text { is anti-phase synchronization. }\end{array}$ \\
\hline 8 & $\begin{array}{l}\Phi_{(2,7)(3,7)} \text { arrives around } 180 \text { degrees, and a phase state between } \\
\text { OSC }(2,7) \text { and } \operatorname{OSC}(3,7) \text { is fixed anti-phase synchronization. }\end{array}$ \\
\hline 9 & $\begin{array}{l}f_{(2,7)} \text { arrives around } f_{\text {anti-anti }} \text {, because the phase state between } \\
\operatorname{OSC}(2,7) \text { and } \operatorname{OSC}(3,7) \text { is anti-phase synchronization. }\end{array}$ \\
\hline
\end{tabular}




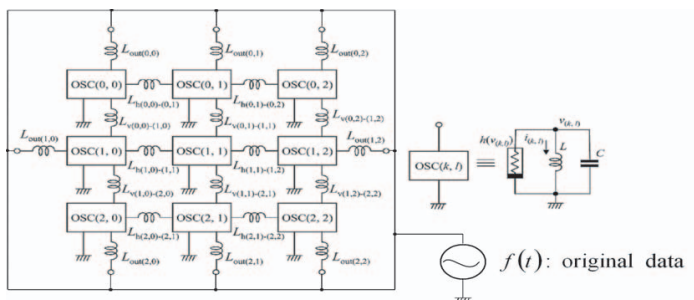

Fig. 6. Circuit model.
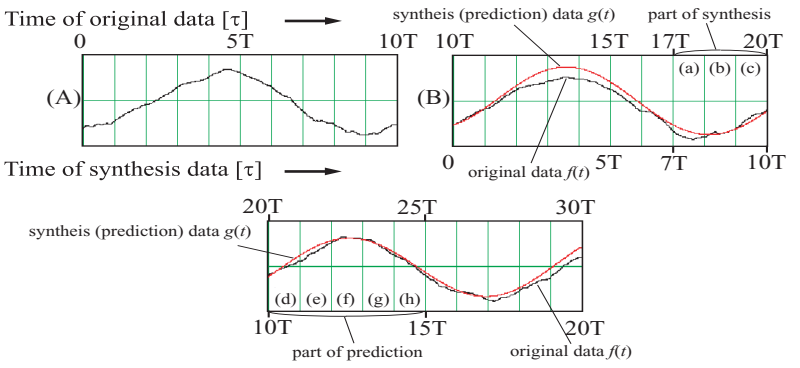

Fig. 7. Original data and synthesis data. (A), (B) and (C) are a continual graph. $0[\tau]$ of original data is set at the left edge of (A). $0[t a u]$ of synthesis data is set at the left edge of (B). Prediction data can be observed in (C).

time period of arbitrary length $T$, and are predicted. We make a system which generates next data from previous data. The next data and the previous data are used known-data. Then, we make future data by using the system and the known-data. The future data are unknown-data. The details of a procedure of our method, that the synthesis (or prediction) time-series data $g(t)$ are made from the original time-series data $f(t)$, are shown as follows: 1. The original data from $f(0)$ to $f(10 T)$ is added to our system, and data from $g(0)$ to $g(10 T)$ is synthesized at the same time. The original data are shown in Fig. 7-(A). The synthesis data are shown in Fig. 7-(B). In the Fig. 7, time axis of synthesis data differs from time axis of original data. 2. The synthesized data $g(t):(7 T \leq t \leq 10 T)$ are adjusted to values close to $f(t):(17 T \leq t \leq 20 \bar{T})$ by changing each parameter and eighteen initial values of oscillators. 3 The original time-series data from $f(10 T)$ to $f(20 T)$ is added to the adjusted system, and time-series data from $g(10 T)$ to $g(20 T)$ is synthesized at the same time. The synthesis data $g(t):(10 T<t \leq 20 T)$ are considered as prediction data. However, to predict for long time is difficult, so a prediction period is fixed from $10 T$ to $15 T$. Then ,if the time-series data $g(t):(10 T<t \leq 15 T)$ is close to $f(t):(20 T<t \leq 25 T)$, we can consider that $g(t):(10 T<t \leq 15 T)$ predicts the original time-series data(see Fig. 7-(C)).

\section{B. Experiment}

In this paper, time-series data of the air temperature in Hiroshima city from April 1981 to May 1985 is used as the original time-series data. Then, $f(t)$ is made by applying the moving average of every 31-day period, and subtracting an average of a maximum and a minimum temperatures of the whole period from each temperature.

In this study, domain (c) is thought to be more important than (a) and domain (b)(see Fig. 7). Therefore, the average of each domain is weighted, and the average error of a synthesisdomain is calculated by the following equation.

$$
\text { synthesiserror }=\frac{e_{a} \times 1+e_{b} \times 1.5+e_{c} \times 2}{3},
$$

where $e_{a}, e_{b}$ and $e_{c}$ express the average errors of domain (a), domain (b) and domain(c) respectively. If the total by

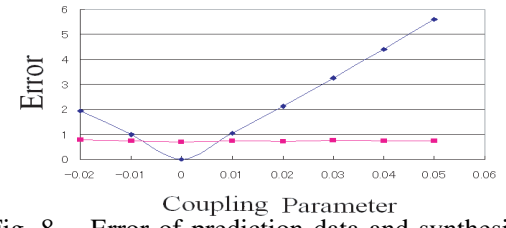

Fig. 8. Error of prediction data and synthesis data.

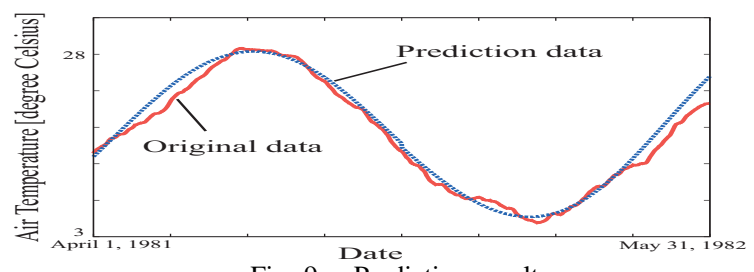

Fig. 9. Prediction result.

the above method is around 0 , good synthesis data is synthesized. We show a relationship between synthesis errors and prediction errors(see Fig.8). The circuit parameters are changed from local optimal solution. We fix some observation condition as follows: 1. Only the coupling parameter of $\operatorname{OSC}(1,1)$ is changed. 2. Averages of errors of prediction data are calculated at the domains (d)-(h). We show the results of errors of prediction data and synthesis data as Fig. 8. In the natural complex time-series data, we can basically observe that prediction error is largely minimum value when synthesis error is minimum value.

The prediction result is shown in Fig. 9. We can observe that the prediction data exists around centerline of original data.

\section{CONCLUSION}

We analyzed and clarified mechanism of propagation of phase-inversion waves on the lattice shape system. The propagation mechanisms of phase-inversion waves, which exist in in-and-in-phase synchronization and in-and-anti-phase synchronization, were explained by the instantaneous frequency of each oscillator and the phase differences of adjacent oscillators. Furthermore, the air temperature data of Hiroshima city was predicted by using the lattice shape system. We made clear that our developed system can be used for prediction of natural complex data.

\section{ACKNOWLEDGEMENTS}

This research is supported by the Grants-in-Aid for Young Scientific Research (B) (No. 19760270) from the Japan Society for the Promotion of Science.

\section{REFERENCES}

[1] L. L. Bonilla, C. J. Pérez Vicente and R. Spigler "Time-Periodic Phases in Populations of Nonlinearly Coupled Oscillators with Bimodal Frequency Distributions," Physica D: Nonlinear Phenomena vol. 113, issues 1, pp. 79-97, Feb. 1998.

[2] C. M. Gray, "Synchronous Oscillations in Neuronal Systems: Mechanisms and Functions," Journal of Computational Neuroscience 1, pp. 11-38, 1994.

[3] T. Endo and S. Mori, "Mode Analysis of Two-Dimensional Low-Pass Multimode Oscillator," IEEE Trans. Circuits and Syst., vol. 23, no. 9, pp. 517-530, Sept. 1976.

[4] M. Yamauchi, Y. Nishio and A. Ushida, "Phase-Waves in a Ladder of Oscillators," IEICE Trans. Fundamentals, vol.E86-A, no.4, pp.891-899, Apr. 2003.

[5] M. Yamauchi, M. Okuda, Y. Nishio and A. Ushida, "Analysis of PhaseInversion Waves in Coupled Oscillators Synchronizing at In-and-AntiPhase," IEICE Trans. Fundamentals, vol.E86-A, no.7, pp.1799-1806, July 2003.

[6] S. Yamane, T. Imoto and M. Yamauchi, "Propagation Mechanism of Phase-Waves on a Lattice of Coupled Oscillators," Proc. SSJW'08, pp.710, Aug. 2008.

[7] S. Yamane, M. Yamauchi and Y. Nishio, "Classification of phenomena on coupled oscillators system as a lattice," Proc. NCSP'09, pp.249-252, Mar. 2009.

[8] T. Imoto, K. Ichiki, S. Yamane and M. Yamauchi, "Phase Difference Propagation Phenomena on a Non-edge Lattice," Proc. NOLTA 2008, pp.616-619, Sept. 2008. 